Research Paper

\title{
CXXC5 Mediates $P$. gingivalis-suppressed Cementoblast Functions Partially via MAPK Signaling Network
}

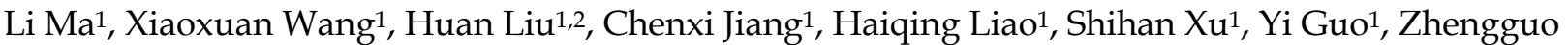 \\ $\mathrm{Cao}^{1,2}{ }^{\boxplus}$
}

1. The State Key Laboratory Breeding Base of Basic Science of Stomatology (Hubei-MOST KLOS) \& Key Laboratory of Oral Biomedicine Ministry of Education (KLOBME), School \& Hospital of Stomatology, Wuhan University, Wuhan, China

2. Department of Periodontology, School \& Hospital of Stomatology, Wuhan University, Wuhan, China

$\square$ Corresponding author: Zhengguo Cao, Department of Periodontology, School \& Hospital of Stomatology, Wuhan University, 237 Luoyu Road, Hongshan District, Wuhan, 430079, China. Tel: +86-27-87686212 Email: caozhengguo@whu.edu.cn

(C) Ivyspring International Publisher. This is an open access article distributed under the terms of the Creative Commons Attribution (CC BY-NC) license (https://creativecommons.org/licenses/by-nc/4.0/). See http://ivyspring.com/terms for full terms and conditions.

Received: 2019.04.02; Accepted: 2019.05.19; Published: 2019.06.04

\begin{abstract}
Porphyromonas $(P$.$) gingivalis associates tightly with periodontal diseases and it is also a dominant$ pathogen of periapical periodontitis. However, the influence of $P$. gingivalis on cementoblasts, root surface cells pivotal in the apical areas, and the possible involvement of other molecules remain largely elusive. CXXC5 is a nuclear protein that regulates gene expression as well as cell growth, differentiation, and apoptosis. In this study, $P$. gingivalis repressed the mineralization capacity of cementoblasts by inducing inflammatory reactions and inhibiting cell differentiation. Intriguingly, the expression of CXXC5 decreased in P. gingivalis-treated OCCM-30 cells and apical periodontitis models but gradually increased during mineralization. Furthermore, RNA interference of CXXC5 significantly inhibited cementoblast differentiation, represented by decline of bone-associated markers Osterix, osteocalcin (OCN), and alkaline phosphatase (ALP). CXXC5 overexpression facilitated differentiation, and therefore attenuated the $P$. gingivalis-repressed effects on OCCM-30 cells. In addition, Erk1/2, p38, and PI3K-Akt were inactivated by silencing CXXC5 and activated upon its overexpression, whereas $\mathrm{Wnt} / \mathrm{\beta}$-catenin exhibited an opposite trend. The employment of specific inhibitors revealed that the CXXC5-dependent promotions of cementoblast differentiation were partially abrogated by $\mathrm{p} 38$ and PI3K-Akt inhibitors but were exacerbated by inhibiting Erk1/2. Overall, our experiment demonstrated a novel function of $\mathrm{CXXC5}$ in the regeneration of impaired cementum caused by $P$. gingivalis invasion and suggested that MAPK signaling network balances the facilitation effects of CXXC5 in cementoblast differentiation.
\end{abstract}

Key words: Porphyromonas gingivalis; cementum; CXXC5; periodontitis; apical periodontitis; signaling regulation

\section{Introduction}

Porphyromonas gingivalis is a Gram-negative oral anaerobe related tightly to periodontitis, which is featured by chronic inflammatory reaction and periodontal tissue destruction [1]. Moreover, $P$. gingivalis is frequently detected on the periapical root surfaces in apical periodontitis [2,3]. Cementoblasts, which are root surface lining cells that share similar characteristics with osteoblasts and coordinate with ambient tissues [4], have limited functions in healthy states but are highly responsive to $P$. gingivalis lipopolysaccharide (LPS) $[5,6]$. In this regard, investigations into how $P$. gingivalis impacts on cementoblast functions and the possible involvement of other molecules are indispensable.

CXXC-type zinc finger protein 5 (CXXC5) is a nuclear protein and functions as a transcription modulator by specific binding with DNA via its CXXC-type zinc-finger domain. This protein is involved in diversified cellular and histological development, including myelopoiesis [7], angiogenesis [8], osteogenesis [9], cardiogenesis [10], and skeletal myogenesis [11]. Moreover, CXXC5 has 
regulatory functions in a wide range of diseases, such as cutaneous wounds [12], hair loss [13], acute myeloid leukemia [14], and hepatocellular carcinoma [15]. The CXXC5 protein has been recognized as a vital coordinator and mediator of Wnt, TGF- $\beta$, BMP, and ATM/p53 pathways [16]. Thus, we wonder whether CXXC5 is related to cementogenesis, a process highly resembles osteogenesis, and the latent molecular mechanisms.

Canonical Wnt/ $\beta$-catenin is the most widely studied signaling in cementoblasts; in general, it suppresses cementoblast differentiation but facilitates proliferation [17]. Mitogen-activated protein kinases (MAPKs), including extracellular signal-regulated kinase (Erk) 1/2, p38, and c-Jun N-terminal kinase (JNK), which mediate various cellular programs, are universally vital for numerous cell types during development [18]. In contrast to Wnt signaling, the role MAPK signaling plays in cementoblasts is obscure, and whether Erk1/2, p38 and JNK exhibit a synergistic effect is also controversial [19-21]. In view of this, scholars need further investigations of MAPKs to broaden our understandings.

Our research aims to clarify the impact of $P$. gingivalis on cementoblast differentiation, the possible involvement of CXXC5 in the process, and the underlying mechanisms. The present paper first verified that CXXC5 attenuates $P$. gingivalis-suppressed cementoblast functions, during which Erk1/2, p38, JNK, PI3K-Akt, and $W n t / \beta$-catenin signaling take effect. Herein, these results indicated CXXC5 may contribute to remodeling of damaged cementum caused by $P$. gingivalis invasion.

\section{Materials and Methods}

\section{Cell Culture and Bacteria Culture}

OCCM-30, a murine cementoblast cell line kindly offered by Dr. M.J. Somerman, was maintained in DMEM (Hyclone) plus 10\% fetal bovine serum (FBS; Every Green) under humidified conditions (5\% $\mathrm{CO}_{2}$ at $37{ }^{\circ} \mathrm{C}$ ) [22]. Osteogenic induction medium (OIM) containing 5\% FBS, $50 \mu \mathrm{g} / \mathrm{mL}$ ascorbic acid (Sigma), and $10 \mathrm{nM} \mathrm{Na} \beta$-glycerophosphate (Sigma) was switched to induce osteogenic differentiation.

$P$. gingivalis (standard strain ATCC 33277) was cultured at $37{ }^{\circ} \mathrm{C}$ in anaerobic incubators $\left(80 \% \mathrm{~N}_{2}, 10 \%\right.$ $\mathrm{H}_{2}$, and $10 \% \mathrm{CO}_{2}$ ), nourished by trypticase soy broth (TSB) with $0.1 \%$ yeast extracts, $1 \mu \mathrm{g} / \mathrm{mL}$ menadione, and $5 \mu \mathrm{g} / \mathrm{mL}$ hemin $(\mathrm{pH}$ 7.46). The bacteria were inoculated from TSB blood agar plates into the liquid medium and went into log phase. A spectrophotometer was applied to determine the concentration at $600 \mathrm{~nm}\left(\mathrm{OD} 1\right.$ equals $10^{9} P$. gingivalis
$/ \mathrm{ml}$ ) [23]. The liquid was centrifuged at low speed, and $P$. gingivalis was resuspended in OCCM-30 growth medium.

\section{Animals and Periapical-lesion Induction}

Male C57BL/6 mice were employed, and periapical lesions were induced as previously described [24], approved by the Ethics Committee of School and Hospital of Stomatology, Wuhan University. 8-week-old mice were anesthetized, permitting straight sight of mandibular first molars. The pulp chambers were unfolded by a No. 1/4 fissure bur until the root canal orifices were clearly seen under a stereomicroscope. The exposed molars were infected with $P$. gingivalis in $2 \%$ carboxymethylcellulose vehicle or not and left open for 14 days ( $\mathrm{n}=$ six mice per group). No surgical interventions were done on the right first molars of the mice without $P$. gingivalis treatment, which were used as the healthy control.

\section{SiRNA Interference, Plasmid Construction, and Transient Transduction}

OCCM-30 cells were inoculated in six-well plates and transiently transfected with si-Cxxc5 (5'-ACA UCU CCA CGU CCC UAG UTT-3' and 5'-ACU AGG GAC GUG GAG AUG UTT-3') or si-NC provided by GenePharma, and the transfection reagent PepMute (Signagen) was used as instructed.

To overexpress the Cxxc5 gene, NC- and Cxxc5-over plasmids were constructed by Miaoling Biotech and transfected into OCCM-30 cells by TurboFect (Thermo Scientific). OIM was applied 8 hours after transduction, and $P$. gingivalis was added together if necessary.

\section{Chemicals and Antibodies}

Specific chemical inhibitors, including PD98059 for Erk 1/2 pathway, SB203580 for p38 pathway, and LY294002 for phosphoinositide-3-kinase (PI3K)-Akt pathway, were purchased from Selleck.

Antibodies for Osterix, OCN, bone sialoprotein (BSP), and $\beta$-catenin were purchased from Abcam. Antibodies for CXXC5, p-Erk1/2, total-Erk1/2, p-p38, total-p38, p-JNK, total-JNK, p-Akt, total-Akt were obtained from Cell Signaling Technology. The $\beta$-actin antibody was purchased from Proteintech.

\section{RNA Isolation and Quantitative Real-Time Polymerase Chain Reaction (qPCR)}

Total RNA was isolated using TRIzol reagent (TaKaRa) and reverse transcribed to first-strand cDNA by PrimeScript RT Reagent Kit with gDNA Eraser (TaKaRa). The Applied Biosystems QuantStudio 6 was used to perform qPCR with SYBR qPCR Master Mix (Vazyme) in triplicate 
(amplification routine: $30 \mathrm{~s}$ at $95^{\circ} \mathrm{C}, 10 \mathrm{~s}$ at $95^{\circ} \mathrm{C}$ for 40 cycles, and $34 \mathrm{~s}$ at $62^{\circ} \mathrm{C}$ ). The primers for mouse Il-6, MCP-1, RANTES, Osx, Bglap, Bsp, Cxxc5 and Gapdh (used as an internal control gene) were synthesized by Sangon Biotech (see Table S1). The relative fold change of gene expression was calculated according to the $2^{-\Delta \Delta C T}$ value and normalized to Gapdh.

\section{Western Blot Analysis}

Confluent cells were harvested using M-PER mammalian protein extraction reagent (Thermo Scientific) and pelleted by high-speed centrifugation at $4{ }^{\circ} \mathrm{C}$ before supernatant collection [25]. A total of $20-40 \mu \mathrm{g}$ protein was separated onto a $10 \%$ or $12 \%$ SDS-PAGE and transferred to polyvinylidene difluoride membranes (Millipore). After 5\% nonfat milk incubation for 1.5 hours, the membranes were immunoblotted separately with primary antibodies against Osterix (1:1000), OCN (1:500), BSP (1:1000), CXXC5 (1:1000), p-Erk1/2 (1:1000), total-Erk1/2 (1:1000), p-p38 (1:1000), total-p38 (1:1000), p-JNK (1:1000), total-JNK (1:1000), p-Akt (1:1000), total-Akt (1:1000), $\beta$-catenin (1:1000), and $\beta$-actin (1:15000; used as the internal control) overnight at $4{ }^{\circ} \mathrm{C}$, followed by the corresponding secondary antibodies for an hour. The membranes were then detected with either the Enhanced Chemiluminescence Detection Reagents (Advansta) or the SuperSignal West Femto Trial Kit (Thermo Scientific) and exposed to the Odyssey LI-COR scanner.

\section{Alkaline Phosphatase (ALP) Staining, ALP Activity Assay, and Alizarin Red Staining}

Cells were inoculated in six-well plates $\left(3 \times 10^{5}\right.$ /well) and cultured in OIM upon sub-confluence. ALP staining was performed on day 4 of mineral induction with NBT/ BCIP (Beyotime), and images were taken. ALP activity assay was achieved by a kit from Nanjing Jiancheng, and data were calculated as instructed.

Cells were treated with $4 \%$ paraformaldehyde and $1 \%$ alizarin red solution (ARS; $\mathrm{pH} 4.2$ ) on day 14 . The mineral nodules were photographed and then dissolved in $10 \%$ cetylpyridinium chloride. Next, the absorbance was determined at $562 \mathrm{~nm}$.

\section{Immunofluorescence staining}

Cells were possessed with $4 \%$ paraformaldehyde and permeabilized by $1 \%$ Triton X-100 for 15 minutes, 24 hours after inoculated onto the slides. The samples were incubated with normal goat serum for 1 hour at $37{ }^{\circ} \mathrm{C}$ and subsequently with rabbit anti-CXXC5 antibodies $(1: 800)$ overnight at $4{ }^{\circ} \mathrm{C}$. Next, the slides were incubated with secondary antibodies conjugated to Cy3 (1:200; Beyotime) for 1 hour, and then with fluorescein isothiocyanate phalloidin (1:200; Yeasen
Biotech) for 40 minutes. The nuclei were dyed with DAPI (ZSGB-BIO) for 2 minutes. A fluorescence microscope was used to observe and to photograph.

\section{Hematoxylin and Eosin (H\&E) Staining and Immunohistochemistry (IHC)}

Mouse mandibles were processed in standard procedures, including formalin fixation, decalcification, and paraffin embedding. The blocks were cut into 5- $\mu \mathrm{m}$ - thick sections, and the mesio-distal serial sections were used for $\mathrm{H} \& \mathrm{E}$ staining. For IHC, the sections were incubated with reagents of UltraSensitive SP IHC Kit (MXB Biotech) as instructed and with primary antibodies anti-Osterix (1:800) and anti-CXXC5 (1:400) overnight at $4{ }^{\circ} \mathrm{C}$. The morphology of the periapical areas and cementoblasts were observed, and images were taken using a microscope.

\section{Statistical Analysis}

All the experiments were performed thrice, separately. Data were analyzed by SPSS 16.0 and GraphPad Prism 8 and expressed as mean \pm SD. First, Shapiro- Wilk and Kolmogorov- Smirnov tests were used to determine the normal distribution. Then, the $t$-test and One-way ANOVA were used to evaluate the statistical differences. Finally, statistical significance was marked with "*" for $\mathrm{P}<0.05$ and “**” for $\mathrm{P}<0.01$.

\section{Results}

\section{$P$. gingivalis induces inflammation and suppresses cementoblast differentiation}

First, when stimulated with $P$. gingivalis (multiplicity of infection [MOI] $=100$ ) for 3, 6, 12, and 24 hours, increased levels of pro-inflammatory cytokines Il-6, MCP-1, and RANTES were observed, with a peak point of 3 hours (Fig. 1A and Fig. S1A). Meanwhile, the levels of osteogenic markers Osx and Bglap decreased in varying degrees (Fig. 1A). Next, $P$. gingivalis with different MOIs $(0,10,100,500)$ were given for 48 hours, the mRNA expression levels of Il-6, MCP-1, and RANTES raised gradually while those of $O s x$ and Bglap fell steadily (Fig. 1B and Fig. S1B). Further, OCCM-30 cells were given OIM and $P$. gingivalis $(\mathrm{MOI}=100)$ treatment for 0,4 , and 7 days, in which the Il-6 expression was enhanced; however, the mRNA and protein levels of Osterix and OCN were declined sharply (Fig. 1C and 1D).

To elucidate $P$. gingivalis stimulation affect cementoblast mineralization in vitro, alizarin red staining was performed after a 2-week mineral induction, which displayed an evident inhibited effect (Fig. 1E). Further semi-quantitative analysis of ARS assays confirmed that mineral nodule formation was 
obviously suppressed by $P$. gingivalis treatment (Fig. 1F). These experiments support the notion that $P$. gingivalis was responsible for the enhanced inflammation and the reduced mineralization in cementoblasts.

\section{CXXC5 increases during differentiation and decreases in $P$. gingivalis-suppressed OCCM-30 cells}

OCCM-30 cells were induced to osteogenic differentiation for $0,4,7$, and 11 days to uncover the expression profile of $\mathrm{CXXC5}$, and it revealed that CXXC5 increased gradually during cementoblast differentiation, along with bone-related markers Osterix and BSP (Fig. 2A and 2B). Interestingly, following $P$. gingivalis infection for different time-lengths or with different MOIs, Cxxc5 mRNA expression levels showed a significant tendency to decline (Fig. 2C and 2D). Further qPCR and western blotting confirmed that CXXC5 was upregulated in cementoblast differentiation process and downregulated under $P$. gingivalis invasion (Fig. 2E and $2 \mathrm{~F}$ ). Immunofluorescence staining revealed that CXXC5 was located in the nucleus of OCCM-30 cells and that after $P$. gingivalis treatment for 0,1 , and 3 hours, the fluorescence signals of CXXC5 dramatically weakened (Fig. 2G). Overall, $P$. gingivalis invasion could induce downregulation of CXXC5 in cementoblasts.
A

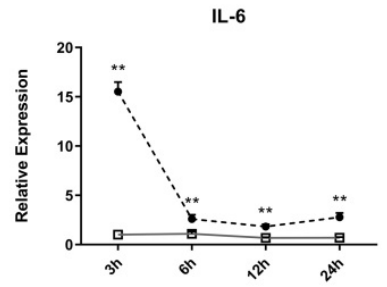

B

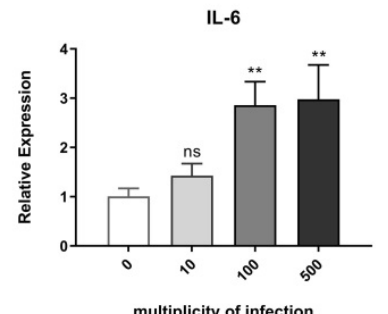

C

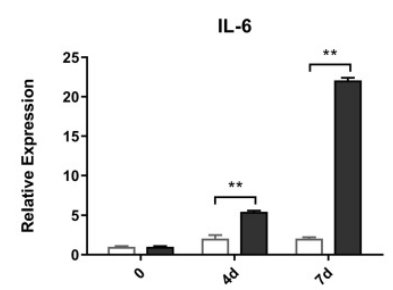

D

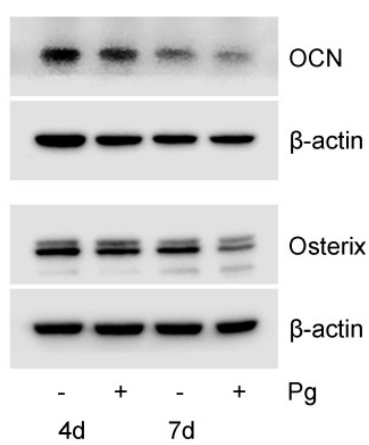

Osx

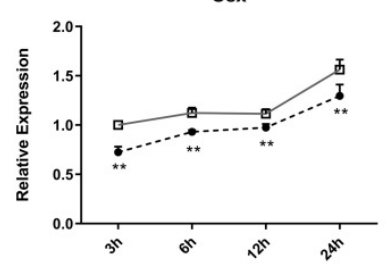

Osx

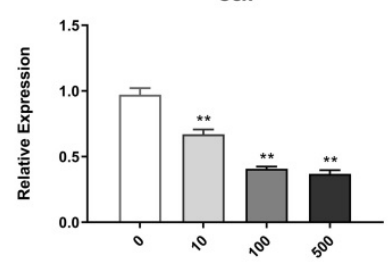

multiplicity of infection

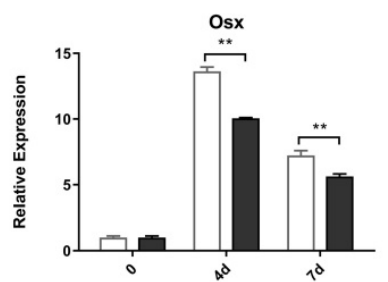

E

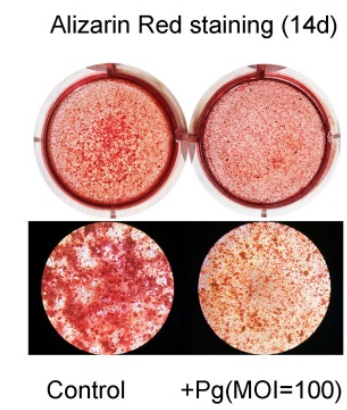

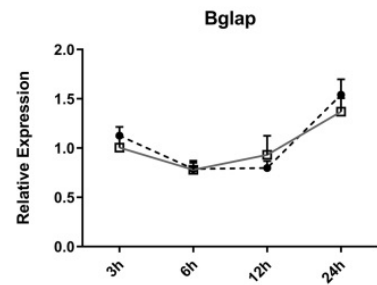

Bglap
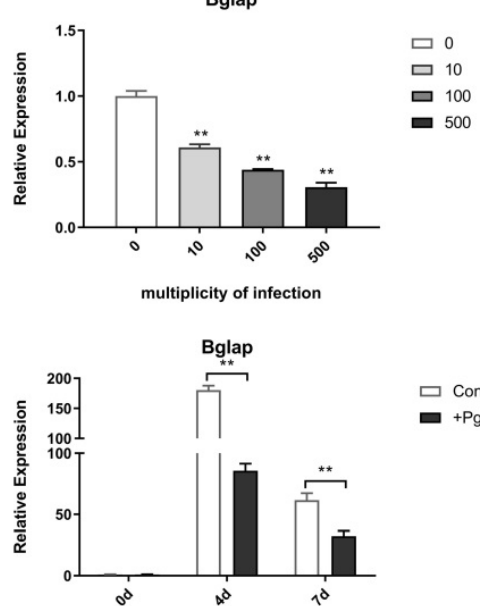

口 Control

ש $+\mathrm{Pg}(\mathrm{MO}=100)$

-. Control

ㅂ. $+\mathrm{Pg}(\mathrm{MOI}=100)$
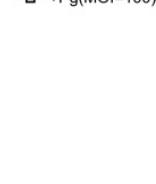

100

500

F

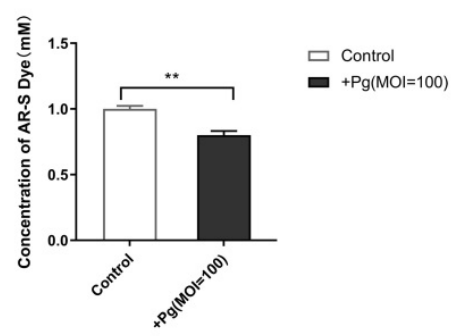

Figure 1. $P$. gingivalis induces inflammation and suppresses mineralization of the OCCM-30 cell line. (A) OCCM-30 was co-cultured with or without $P$. gingivalis for $3,6,12$, and 24 hours. II-6, Osx, and Bglap levels were examined by QPCR. *** for intergroup difference between control and $P$. gingivalis treatment at the observing time-points. (B) II-6 expression was elevated while Osx and Bglap expressions were lowered with different $P$. gingivalis concentrations after a 48-hour osteogenic induction. The MOI 0 group was set as the control. (C, D) OCCM-30 with osteogenic induction and a simultaneous $P$. gingivalis co-culture for 0,4 , and 7 days. II-6, Osx, and Bglap expressions were revealed by qPCR and Western blot analysis. (E, F) OCCM-30 with continuous $P$. gingivalis treatment or not were analyzed with ARS on day 14 . $10 \%$ cetylpyridinium chloride was applied to dissolve mineral nodules, and the absorbance was determined at $562 \mathrm{~nm}$. Pg, P. gingivalis. Gapdh or $\beta$-actin was adopted as an internal reference. The values are presented as mean $\pm \mathrm{SD}, * \mathrm{P}<0.05$, and $* * \mathrm{P}<0.01$. 
A

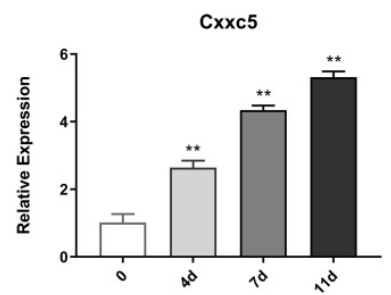

B

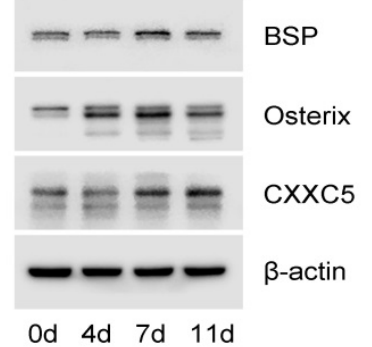

E

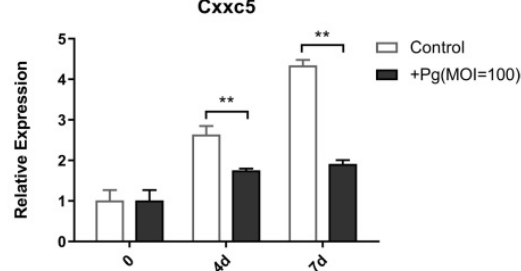

F

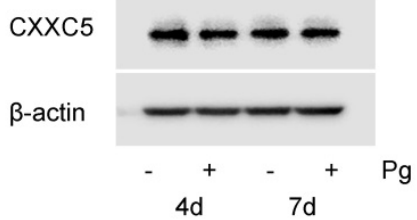

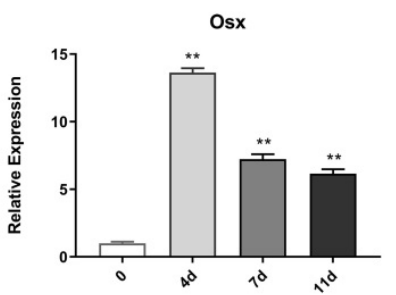

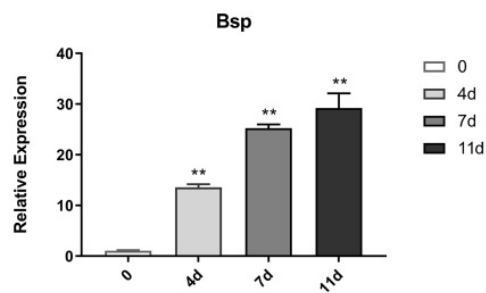

C
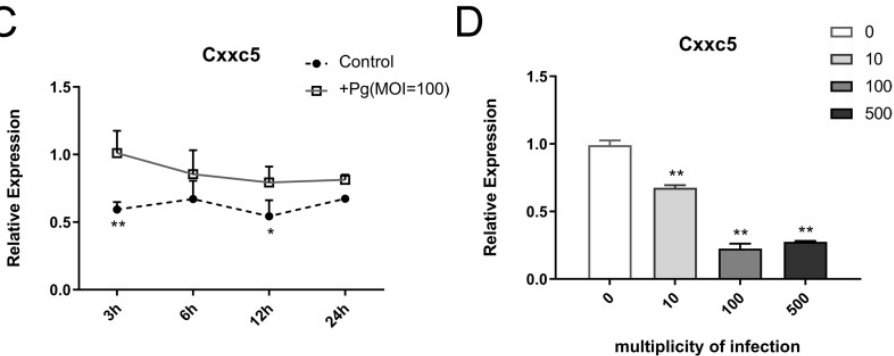

G

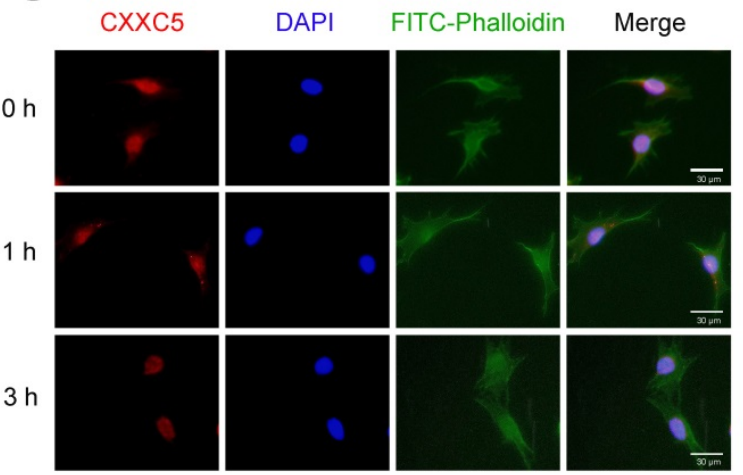

Figure 2. CXXC5 increases during differentiation and decreases in $\mathbf{P}$. gingivalis-suppressed OCCM-30 cells. (A, B) The CXXC5 expression level was upregulated during cementoblast differentiation, together with Osterix and BSP, examined by QPCR and Western blot analysis. The day 0 group was set as the control. (C) OCCM-30 was co-cultured with or without $P$. gingivalis for $3,6,12$, and 24 hours. $C_{x x c 5}$ expression level was determined by qPCR. * and $* *$ for intergroup difference between control and $P$. gingivalis treatment at the observing time-points. (D) $C x x c 5$ expression decreased with different $P$. gingivalis concentrations after a 48 -hour osteogenic induction. The MOI 0 group was set as the control. (E, F) OCCM-30 with osteogenic induction and simultaneous $P$. gingivalis co-culture for 0,4 , and 7 days. CXXC5 expression was revealed by qPCR and Western blot analysis. (G) Immunofluorescence of CXXC5 signals weakened in OCCM-30 cells after $P$. gingivalis stimulation for the indicated times. Pg, $P$. gingivalis. Gapdh or $\beta$-actin was adopted as an internal reference. The values are presented as mean \pm SD, $* P<0.05$, and $* * P<0.01$.

\section{CXXC5 declines in $P$. gingivalis-aggravated apical periodontitis in vivo}

As previously described, P. gingivalis was detected in the cementum-attached plaque region of periodontal lesions [26], as well as on the periapical root surfaces of apical lesions [2]. However, compared with periodontitis models wherein epithelial barrier allowed traces of bacteria to interact with interior cementoblasts, $P$. gingivalis mass was observed in apical areas when applied through root canal system [27]. Consequently, we chose to establish apical periodontitis (AP) models.

We set up the periapical periodontitis murine model successfully, where inflammatory cell accumulation could be clearly observed by $\mathrm{H} \& \mathrm{E}$ staining on day 14 (Fig. 3A). IHC staining showed that Osterix and $\mathrm{CXXC5}$ were positive in cementoblast cells and ambient periodontal ligament cells. As expected, it showed a sharply reduced expression of Osterix and a slightly reduced expression of CXXC5 in cementoblasts in the following three groups: the healthy group, the AP group and the AP plus $P$. gingivalis group (Fig. 3B and $3 \mathrm{C}$ ). The black arrows in the pictures indicate inflammatory cells and the red arrows indicate cementoblasts.

In summary, the in vitro and in vivo results forecast that CXXC5 may be indispensable in the regulation of cementoblast differentiation and mineralization.

\section{CXXC5 mediates cementoblast differentiation, and CXXC5 overexpression partially attenuates $P$. gingivalis-suppressed effects}

To further explore the biological functions of CXXC5 during cementoblast differentiation, siRNA and plasmids were applied to obtain knockdown and 
overexpression effects, respectively. After si-NC and si-Cxxc5 were transfected for 24 hours, the cell growth medium was changed to OIM. Data displayed that the Osterix and OCN expression levels were downregulated on days 2 and 4, whereas CXXC5 showed a stable silence efficiency of approximately five folds (Fig. 4A and 4B). Similarly, after the NCand the Cxxc5-over plasmids were transfected, the medium was changed with OIM containing $P$. gingivalis $(\mathrm{MOI}=0$ or 100$)$. $\mathrm{qPCR}$ and Western bot analysis demonstrated that the CXXC5, Osterix and OCN expression levels were mitigated under $P$. gingivalis treatment and reversed by CXXC5 overexpression (Fig. 4E and 4F). Moreover, ALP activity assay and ALP staining confirmed that CXXC5 mediated the mineralization ability of cementoblasts (Fig. 4C, 4D, 4G, and 4H).
A

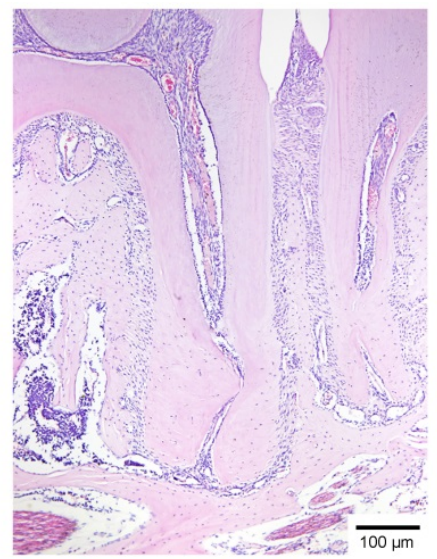

B

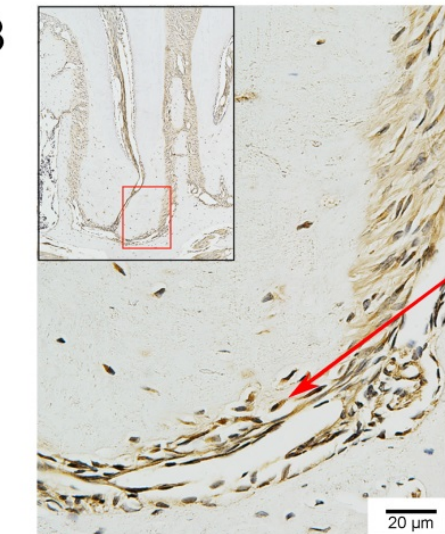

Healthy

C

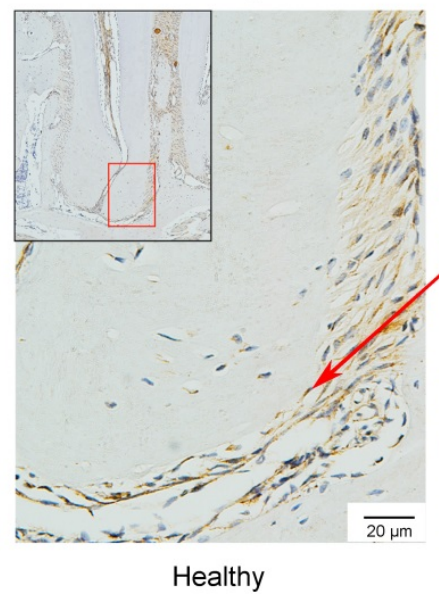

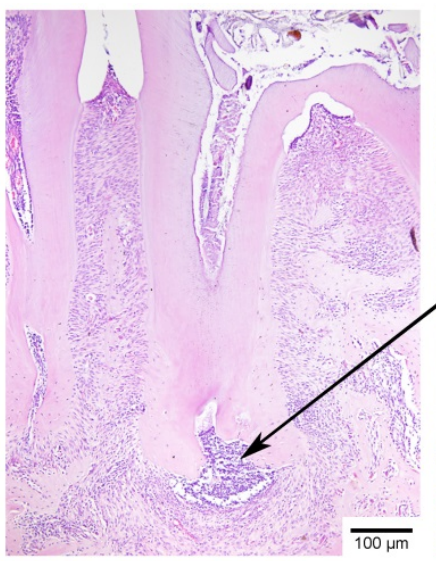

Apical periodontitis

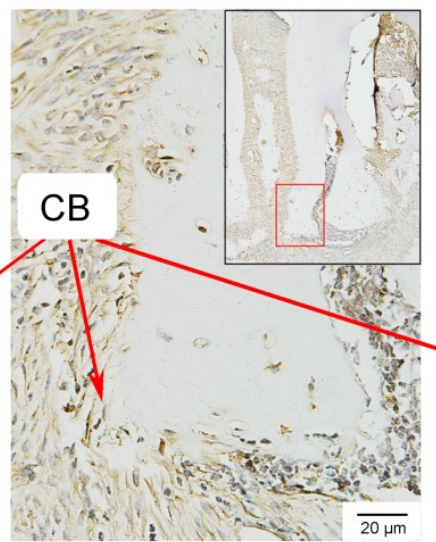

Apical periodontitis

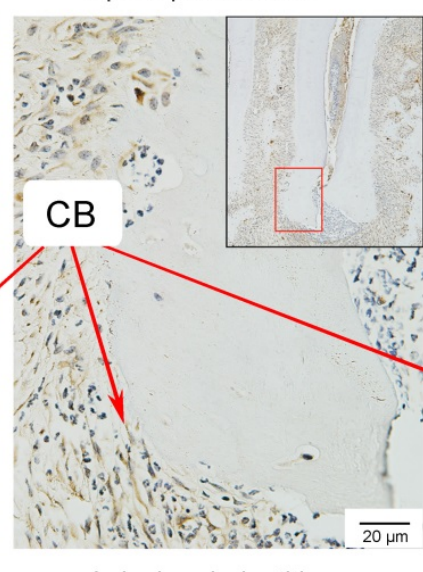

Apical periodontitis

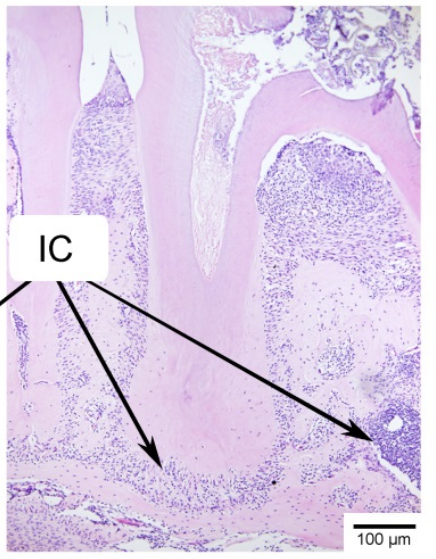

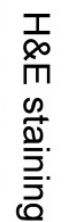

Apical periodontitis $+\mathrm{Pg}$

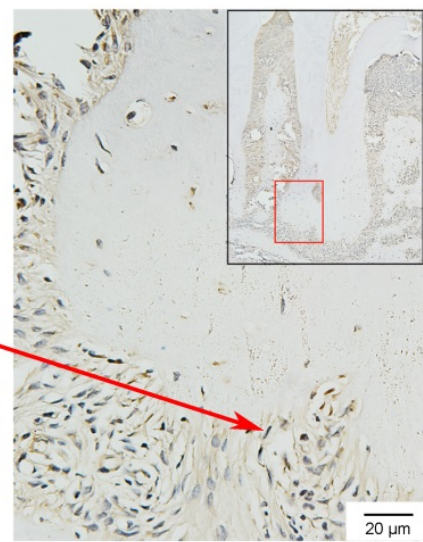

$\stackrel{\bigcirc}{C}$
$\frac{\mathbb{D}}{\bar{x}}$
Apical periodontitis $+\mathrm{Pg}$

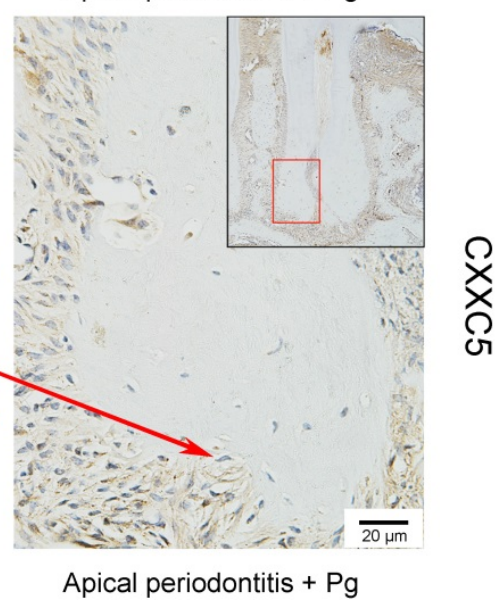

Figure 3. CXXC5 declines in P. gingivalis-aggravated apical periodontitis in vivo. (A) H\&E staining showed the successfully established periapical periodontitis murine model and the inflammatory cell infiltration on day 14. (B) IHC staining displayed the expression of Osterix in the periapical areas of healthy and of AP-induced mice (with or without $P$. gingivalis invasion). (C) IHC staining displayed the expression of CXXC5 in the periapical areas of healthy and of AP-induced mice (with or without $P$. gingivalis invasion). The black arrows indicate inflammatory cells, and the red arrows indicated cementoblasts. $\mathrm{Pg}, P$. gingivalis. 
A

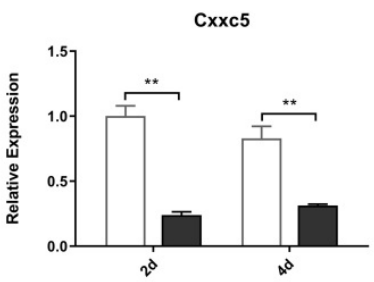

B

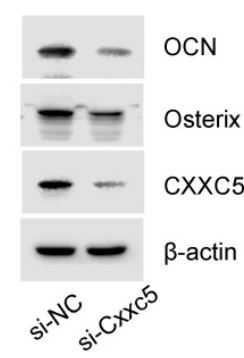

E

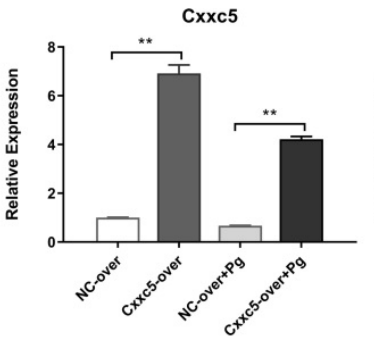

G

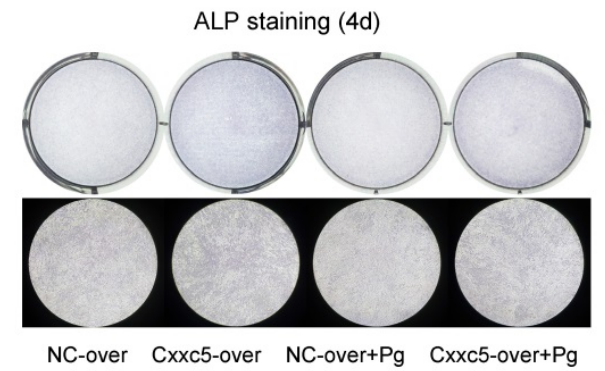

C

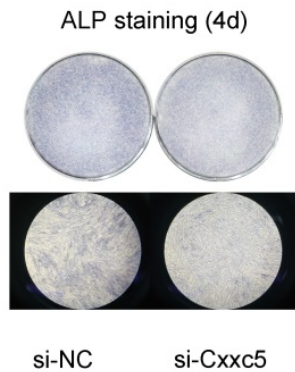

Bglap

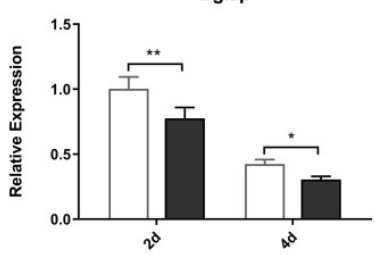

口 si-NC

si-Cxxc5
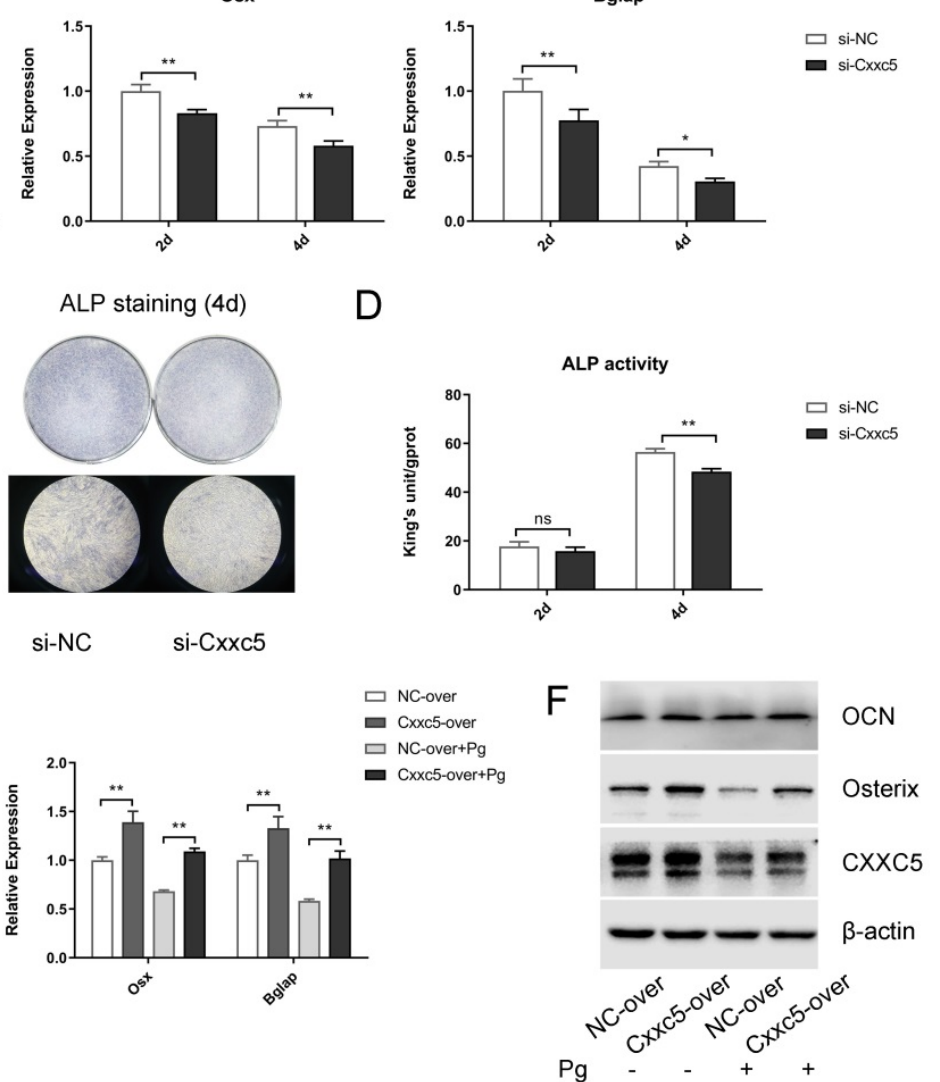

$\mathrm{H}$

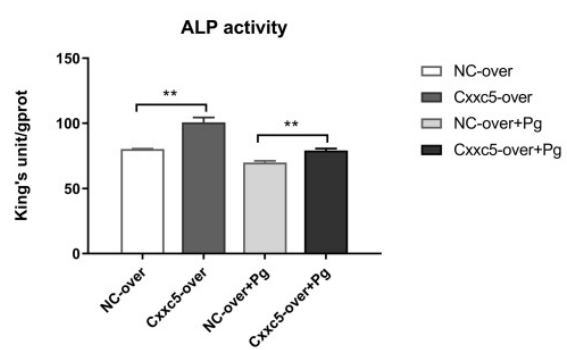

Figure 4. CXXC5 knockdown suppresses cementoblast differentiation, and CXXC5 overexpression partially attenuates $P$. gingivalis-suppressed effects. (A, B) Osterix and OCN expression levels were downregulated with CXXC5 under RNA interference and simultaneous OIM on days 2 and 4, examined with qPCR and Western blot analysis. (C, D) ALP activity assay and ALP staining on days 2 and 4, respectively, proved the impaired mineralization ability of cementoblasts with silenced CXXC5. (E, F) The CXXC5, Osterix, and OCN expression levels were mitigated under $P$. gingivalis treatment but reversed by $C X X C 5$ overexpression, examined with $\mathrm{QPCR}$ and Western blot analysis. (G, H) ALP activity assay and ALP staining on day 4, proved that impaired mineralization caused by $P$. gingivalis was attenuated by $C X X C 5$ overexpression. $\mathrm{Pg}, P$. gingivalis. Gapdh or $\beta$-actin was adopted as an internal reference. The values are presented as mean $\pm \mathrm{SD}, * \mathrm{P}<0.05$, and $* * \mathrm{P}<0.01$.

\section{CXXC5 mediates cementoblast}

\section{differentiation via complicated signaling} network

Next, we evaluated five different signaling pathways to explore the potential mechanisms. Among them, p-Erk1/2, p-p38, and p-Akt were significantly suppressed whereas $\beta$-catenin was obviously activated after CXXC5 silence, and a relatively stable p-JNK/ total JNK ratio was showed, compared with those in the si-NC group (Fig. 5A). The opposite results were achieved in the overexpression group (Fig. 5B), suggesting that MAPKs, PI3K-Akt, and Wnt may be vital during
CXXC5-mediated cementoblast differentiation.

To validate whether CXXC5-dependent promotion of differentiation was achieved via these signals, we applied specific inhibitors of activated pathways under CXXC5 overexpression. The expression levels of Osterix, OCN, and BSP were examined (Fig. 5C and 5D), which indicated that the facilitation effects of CXXC5 were partially abrogated by p38 and PI3K-Akt inhibitors but were exacerbated by inhibiting Erk1/2 pathway. Herein, three common signals making up MAPKs network, exhibited contrasting and balanced effects on CXXC5-promoted cementoblast differentiation. 
A

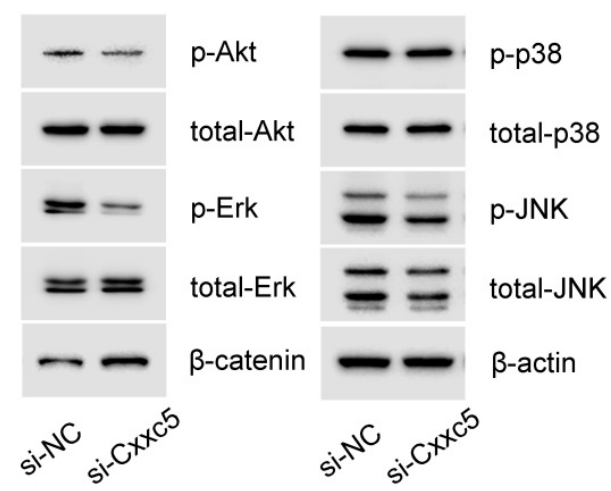

B

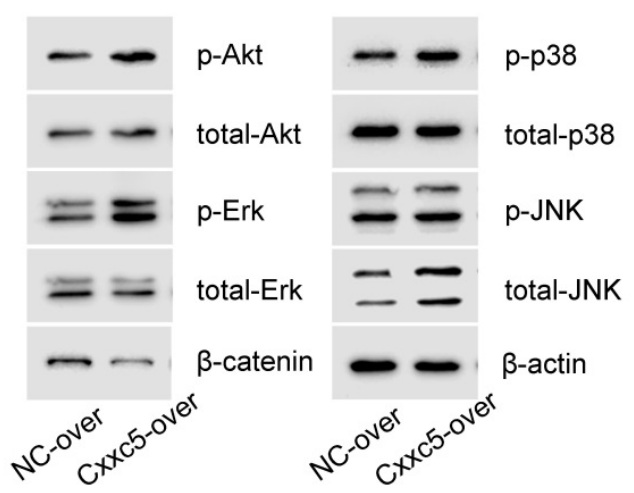

D

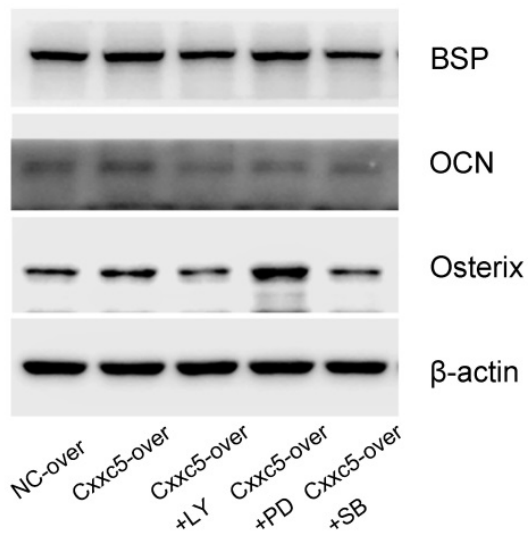

Figure 5. CXXC5 mediates cementoblast differentiation via complicated signaling network. (A) Erk1/2, p38, and PI3K-Akt were inactivated in varying degrees and $\beta$-catenin was significantly activated during the downregulation of $C X X C 5$, shown by Western blot analysis. (B) Erk1/2, p38, and PI3K-Akt were activated and $\beta$-catenin was inactivated during CXXC5 upregulation, shown by Western blot analysis. (C, D) The Osterix, OCN, and BSP expression levels were enhanced under CXXC5 forced expression but reversed by $\mathrm{P} 38$ and PI3K-Akt pathway inhibitors, examined with qPCR and Western blot analysis. LY, LY294002, PI3K-Akt pathway inhibitor; PD, PD98059, Erk1/2 pathway inhibitor; SB, SB203580, 38 pathway inhibitor. Gapdh or $\beta$-actin was adopted as an internal reference. The values are presented as mean \pm SD, $* \mathrm{P}<0.05$, and $* * \mathrm{P}<0.01$ compared with the Cxxc5-over+DMSO group.

\section{Discussion}

In our study, CXXC5 was regarded as a novel mediator of $P$. gingivalis-repressed cementoblast differentiation and mineralization. This research indicated that CXXC5 affected cementoblast functions, with MAPKs, PI3K-Akt, and Wnt signaling pathways involved (Fig. 6). Overall, this study suggested that overexpressing CXXC5 may be conducive in impaired cementum regeneration after $P$. gingivalis invasion.

Cementum, particularly cellular cementum with imbedded cementocytes, is a crucial part in the formation, repair, and regeneration of the periodontium. The bone-like structure covering the root serves as a solid barrier against oral bacteria invasion [28]. Cementoblasts, root lining cells, share similar features with osteoblasts, such as the expression of bone-associated markers, including Osterix, OCN, and BSP [29]. Previous studies by our group manifested many molecules generated during cementogenesis like osteogenic markers and microRNAs are beneficial for regeneration [25, 30-32]. Therefore, obtaining insights into tissue regeneration requires further understanding on the reconstruction of impaired cementum upon bacterial invasion.

In this study, $P$. gingivalis, rather than its components, such as LPS, was employed as a stimulating factor to cementoblasts. As a premise, $P$. gingivalis was detected deep in the cementum-attached plaque region of periodontal pockets and on the periapical root surfaces of apical lesions $[2,26]$. Previous reports have exhibitd that $P$. gingivalis LPS regulates cementoblast functions, and thereby affects the inflammation-induced resorption of cementum $[5,6]$. However, $P$. gingivalis consists of multiple pathogenic ingredients [33]. For instance, the presence of fimbriae greatly enhances adhesivity and aggressivity [34, 35]. Moreover, secreted gingipains notably modulates inflammatory cytokine production and thus causes destruction [36,37]. Taken together, the total bacteria treatment could fully reveal its functional advantages and simulate pathological processes in vivo. In addition, different strains of bacteria caused damages ranging from small, localized abscesses to invasive, spreading infections [38]. 


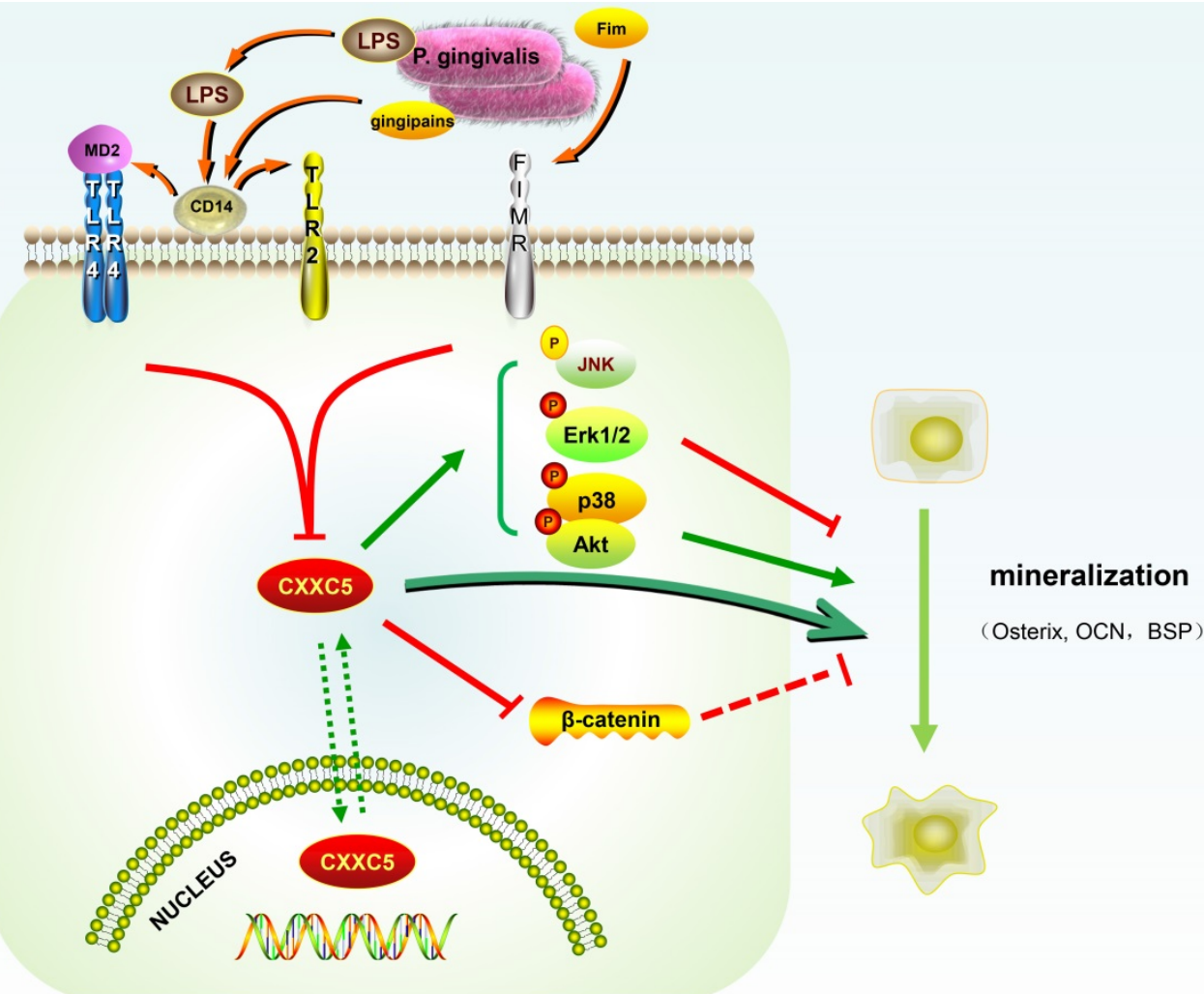

Figure 6. Schematic illustration. CXXC5 mediates $P$. gingivalis-suppressed cementoblast differentiation and mineralization, with MAPKs, PI3K-Akt, and Wnt signaling pathways involved. LPS: lipopolysaccharide; Fim: fimbriae; MD2: myeloid differentiation 2; CD14: cluster differentiation 14; TLR2: Toll-like receptor 2; TLR4: Toll-like receptor 4; FIMR: fimbrillin receptor.

Our experiment showed that $P$. gingivalis induced inflammatory responses in OCCM-30 cells and in animal models. Afterwards, the capacities of cementoblast to differentiate and mineralize were suppressed in this environment. However, the relevance of single virulence factor to cementoblast functions and their possible contribution to cementum impairment remain obscure.

CXXC-type zinc finger protein 5, though short in length (322 amino acids), is capable of binding to DNA and serving as a regulator and coordinator of complex signaling pathways [16]. This protein modulates gene expression, cell proliferation, differentiation, and apoptosis in various cell types $[8$, $9,11,39]$. Abnormal expression or activity of CXXC5 also results in immune response disorders [40, 41]. Intriguingly, previous studies have summarized that CXXC5 was involved in a sophisticated signaling network, being conducted while simutaneously regulating others $[9,10,15,42]$. Among those signaling transduction processes, the $\mathrm{Wnt} / \beta$-catenin signaling is closely correlated with and negatively regulated by CXXC5 [43] . In summary, we attempted to explore the precise functions of CXXC5 in cementoblasts under inflammatory stimulation and osteogenic induction and to further clarify its potential mechanisms.
In this study, CXXC5 displayed an expression pattern of a steady increase in cementoblasts similar with those in myoblasts and osteoblasts during differentiation $[9,11]$. Furthermore, RNAi interference inhibited expressions of Osterix and OCN and ameliorated ALP activity while CXXC5 overexpression facilitated osteogenic differentiation and thereby attenuated the negative effects $P$. gingivalis exerted on cementoblasts. These findings indicate that CXXC5 plays an active part in $P$. gingivalis-induced inflammation and $P$. gingivalis-inhibited mineralization.

A total of five regular cell signaling profiles were evaluated as Erk1/2, p38, JNK, PI3K-Akt, and Wnt $/ \beta$-catenin as the underlying mechanisms. As expected, $\beta$-catenin presented an opposite changing tendency to CXXC5, which confirmed CXXC5 served as a negative mediator of Wnt [43]. It is generally accepted that Wnt inhibit cementoblast differentiation [17], which makes sense in this situation. In addition, compared with control group, the other four vital signals were inactivated when CXXC5 was silenced and activated when CXXC5 was overexpressed separately. Those pathways are important for cell proliferation and differentiation; however, whether CXXC5 influenced cementoblast differentiation via all of them remains unsettled. Further investigation, such 
as the adoption of inhibitors or activators for more signaling pathways, is necessary to obtain a precise answer.

Our study demonstrated that CXXC5 enhanced the osteogenic differentiation of cementoblasts and thus partially attenuated the $P$. gingivalis-repressed effect, with implicated Erk1/2, p38, JNK, PI3K-Akt and $W n t / \beta$-catenin signaling. These results reflect that CXXC5 functions as a positive modulator of $P$. gingivalis-suppressed cementoblast differentiation. CXXC5 may be effective as a therapeutic target for impaired cementum reconstruction and thus may achieve periodontium regeneration.

\section{Abbreviations}

P. gingivalis: Porphyromonas gingivalis; CXXC5: CXXC-type zinc finger protein 5; MAPKs: Mitogen-activated protein kinases; Erk: extracellular signal-regulated kinase; JNK: c-Jun N-terminal kinase; PI3K: phosphoinositide-3-kinase; LPS: lipopolysaccharide; Fim: fimbriae; MD2: myeloid differentiation 2; CD14: cluster differentiation 14; TLR2: Toll-like receptor 2; TLR4: Toll-like receptor 4; FIMR: fimbrillin receptor.

\section{Supplementary Material}

Supplementary figures and tables. http://www.ijbs.com/v15p1685s1.pdf

\section{Acknowledgments}

The study was supported by grants from the National Natural Science Foundation of China to Zhengguo Cao (No. 81870776 and No. 81570946).

\section{Competing Interests}

The authors have declared that no competing interest exists.

\section{References}

1. Rodriguez-Pato RB. Root resorption in chronic periodontitis: a morphometrical study. J Periodontol. 2004; 75: 1027-1032.

2. Ricucci D, Siqueira JF, Jr. Biofilms and apical periodontitis: study of prevalence and association with clinical and histopathologic findings. J Endod. 2010; 36: 1277-1288.

3. Kitano T, Mikami Y, Iwase T, Asano M, Komiyama K. Loop-mediated isothermal amplification combined with PCR and immunohistochemistry for detecting Porphyromonas gingivalis in periapical periodontitis. J Oral Sci. 2016; 58: 163-169.

4. Bosshardt DD. Are cementoblasts a subpopulation of osteoblasts or a unique phenotype? J Dent Res. 2005; 84: 390-406.

5. Nociti FH, Jr., Foster BL, Barros SP, Darveau RP, Somerman MJ. Cementoblast gene expression is regulated by Porphyromonas gingivalis lipopolysaccharide partially via toll-like receptor-4/MD-2. J Dent Res. 2004; 83: 602-607.

6. Nemoto E, Darveau RP, Foster BL, Nogueira-Filho GR, Somerman MJ. Regulation of cementoblast function by P. gingivalis lipopolysaccharide via TLR2. J Dent Res. 2006; 85: 733-738.

7. Pendino F, Nguyen E, Jonassen I, Dysvik B, Azouz A, Lanotte M, et al. Functional involvement of RINF, retinoid-inducible nuclear factor (CXXC5), in normal and tumoral human myelopoiesis. Blood. 2009; 113: 3172-3181.

8. Kim HY, Yang DH, Shin SW, Kim MY, Yoon JH, Kim S, et al. CXXC5 is a transcriptional activator of Flk-1 and mediates bone morphogenic protein-induced endothelial cell differentiation and vessel formation. FASEB J. 2014; 28: 615-626.
9. Kim HY, Yoon JY, Yun JH, Cho KW, Lee SH, Rhee YM, et al. CXXC5 is a negative-feedback regulator of the Wnt/beta-catenin pathway involved in osteoblast differentiation. Cell Death Differ. 2015; 22: 912-920.

10. Peng X, Li G, Wang Y, Zhuang J, Luo R, Chen J, et al. CXXC5 is required for cardiac looping relating to TGFbeta signaling pathway in zebrafish. Int $\mathrm{J}$ Cardiol. 2016; 214: 246-253.

11. Li G, Ye X, Peng X, Deng Y, Yuan W, Li Y, et al. CXXC5 regulates differentiation of $\mathrm{C} 2 \mathrm{C} 12$ myoblasts into myocytes. J Muscle Res Cell Motil. 2014; 35: 259-265.

12. Lee SH, Kim MY, Kim HY, Lee YM, Kim H, Nam KA, et al. The Dishevelled-binding protein CXXC5 negatively regulates cutaneous wound healing. J Exp Med. 2015; 212: 1061-1080.

13. Lee SH, Seo SH, Lee DH, Pi LQ, Lee WS, Choi KY. Targeting of CXXC5 by a Competing Peptide Stimulates Hair Regrowth and Wound-Induced Hair Neogenesis. J Invest Dermatol. 2017; 137: 2260-2269.

14. Kuhnl A, Valk PJ, Sanders MA, Ivey A, Hills RK, Mills KI, et al. Downregulation of the Wnt inhibitor CXXC5 predicts a better prognosis in acute myeloid leukemia. Blood. 2015; 125: 2985-2994.

15. Yan X, Wu J, Jiang Q, Cheng H, Han JJ, Chen YG. CXXC5 suppresses hepatocellular carcinoma by promoting TGF-beta-induced cell cycle arrest and apoptosis. J Mol Cell Biol. 2018; 10: 48-59.

16. Xiong $\mathrm{X}, \mathrm{Tu} \mathrm{S}$, Wang J, Luo S, Yan X. CXXC5: A novel regulator and coordinator of TGF-beta, BMP and Wnt signaling. J Cell Mol Med. 2019; 23: 740-749.

17. Nemoto E, Koshikawa Y, Kanaya S, Tsuchiya M, Tamura M, Somerman MJ, et al. Wnt signaling inhibits cementoblast differentiation and promotes proliferation. Bone. 2009; 44: 805-812.

18. Cargnello M, Roux PP. Activation and function of the MAPKs and their substrates, the MAPK-activated protein kinases. Microbiol Mol Biol Rev. 2011; 75: 50-83.

19. Kanaya S, Komatsu H, Shimauchi H, Nemoto E. Metabotropic glutamate receptor 1 promotes cementoblast proliferation via MAP kinase signaling pathways. Connect Tissue Res. 2016; 57: 417-426.

20. Kajiya M, Takeshita K, Kittaka M, Matsuda S, Ouhara K, Takeda K, et al. BDNF mimetic compound LM22A-4 regulates cementoblast differentiation via the TrkB-ERK/Akt signaling cascade. Int Immunopharmacol. 2014; 19: 245-252.

21. Sanchavanakit N, Saengtong W, Manokawinchoke J, Pavasant P. TNF-alpha stimulates MMP-3 production via PGE2 signalling through the NF-kB and p38 MAPK pathway in a murine cementoblast cell line. Arch Oral Biol. 2015; 60: 1066-1074.

22. D'Errico JA, Berry JE, Ouyang H, Strayhorn CL, Windle JJ, Somerman MJ. Employing a transgenic animal model to obtain cementoblasts in vitro. J Periodontol. 2000; 71: 63-72.

23. Zhang $\mathrm{W}, \mathrm{Ju}$ J, Rigney $\mathrm{T}$, Tribble G. Porphyromonas gingivalis infection increases osteoclastic bone resorption and osteoblastic bone formation in a periodontitis mouse model. BMC Oral Health. 2014; 14: 89.

24. Wu Y, Sun H, Yang B, Liu X, Wang J. 5-Lipoxygenase Knockout Aggravated Apical Periodontitis in a Murine Model. J Dent Res. 2018; 97: 442-450.

25. Wang X, Sun H, Liao H, Wang C, Jiang C, Zhang Y, et al. MicroRNA-155-3p Mediates TNF-alpha-Inhibited Cementoblast Differentiation. J Dent Res. 2017; 96: 1430-1437.

26. Noiri Y, Li L, Yoshimura F, Ebisu S. Localization of Porphyromonas gingivalis-carrying fimbriae in situ in human periodontal pockets. J Dent Res. 2004; 83: 941-945.

27. Ao M, Miyauchi M, Inubushi T, Kitagawa M, Furusho H, Ando T, et al. Infection with Porphyromonas gingivalis exacerbates endothelial injury in obese mice. PLoS One. 2014; 9: e110519.

28. Arzate H, Zeichner-David M, Mercado-Celis G. Cementum proteins: role in cementogenesis, biomineralization, periodontium formation and regeneration. Periodontol 2000. 2015; 67: 211-233.

29. D'Errico JA, MacNeil RL, Takata T, Berry J, Strayhorn C, Somerman MJ. Expression of bone associated markers by tooth root lining cells, in situ and in vitro. Bone. 1997; 20: 117-126.

30. Cao Z, Zhang H, Zhou X, Han X, Ren Y, Gao T, et al. Genetic evidence for the vital function of Osterix in cementogenesis. J Bone Miner Res. 2012; 27: 1080-1092.

31. Cao Z, Liu R, Zhang H, Liao H, Zhang Y, Hinton RJ, et al. Osterix controls cementoblast differentiation through downregulation of Wnt-signaling via enhancing DKK1 expression. Int J Biol Sci. 2015; 11: 335-344.

32. Wang C, Liao H, Sun H, Zhang Y, Cao Z. MicroRNA-3064-3p regulates the differentiation of cementoblasts through targeting DKK1. J Periodontal Res. 2018; 53: 705-713.

33. Nakayama M, Ohara N. Molecular mechanisms of Porphyromonas gingivalis-host cell interaction on periodontal diseases. Jpn Dent Sci Rev. 2017; 53: $134-140$

34. Mantri CK, Chen CH, Dong X, Goodwin JS, Pratap S, Paromov V, et al. Fimbriae-mediated outer membrane vesicle production and invasion of Porphyromonas gingivalis. Microbiologyopen. 2015; 4: 53-65.

35. Amano A, Fujiwara T, Nagata H, Kuboniwa M, Sharma A, Sojar HT, et al. Prophyromonas gingivalis fimbriae mediate coaggregation with Streptococcus oralis through specific domains. J Dent Res. 1997; 76: 852-857.

36. Bengtsson T, Khalaf A, Khalaf H. Secreted gingipains from Porphyromonas gingivalis colonies exert potent immunomodulatory effects on human gingival fibroblasts. Microbiol Res. 2015; 178: 18-26. 
37. Klarstrom Engstrom K, Khalaf H, Kalvegren H, Bengtsson T. The role of Porphyromonas gingivalis gingipains in platelet activation and innate immune modulation. Mol Oral Microbiol. 2015; 30: 62-73.

38. Sundqvist G, Figdor D, Hanstrom L, Sorlin S, Sandstrom G. Phagocytosis and virulence of different strains of Porphyromonas gingivalis. Scand J Dent Res. 1991; 99: 117-129.

39. Wang X, Liao P, Fan X, Wan Y, Wang Y, Li Y, et al. CXXC5 Associates with Smads to Mediate TNF-alpha Induced Apoptosis. Curr Mol Med. 2013; 13: 1385-1396.

40. Ma S, Wan X, Deng Z, Shi L, Hao C, Zhou Z, et al. Epigenetic regulator CXXC5 recruits DNA demethylase Tet2 to regulate TLR7/9-elicited IFN response in pDCs. J Exp Med. 2017; 214: 1471-1491.

41. Tsuchiya Y, Naito T, Tenno M, Maruyama M, Koseki H, Taniuchi I, et al. ThPOK represses CXXC5, which induces methylation of histone $\mathrm{H} 3$ lysine 9 in Cd40lg promoter by association with SUV39H1: implications in repression of CD40L expression in CD8+ cytotoxic T cells. J Leukoc Biol. 2016; 100: 327-338.

42. Andersson T, Sodersten E, Duckworth JK, Cascante A, Fritz N, Sacchetti P, et al. CXXC5 is a novel BMP4-regulated modulator of Wnt signaling in neural stem cells. J Biol Chem. 2009; 284: 3672-3681.

43. Kim D, Garza LA. The Negative Regulator CXXC5: Making WNT Look a Little Less Dishevelled. J Invest Dermatol. 2017; 137: 2248-2250. 